UCLA/04/TEP/07

hep-th/0404073

\title{
Splitting hairs of the three charge black hole.
}

\author{
Iosif Bena \\ Department of Physics and Astronomy \\ University of California \\ Los Angeles, CA 90095, USA \\ iosif@physics.ucla.edu
}

\begin{abstract}
We construct the large radius limit of the metric of three charge supertubes and three charge BPS black rings by using the fact that supertubes preserve the same supersymmetries as their component branes. Our solutions reproduce a few of the properties of three charge supertubes found recently using the Born Infeld description. Moreover, we find that these solutions pass a number of rather nontrivial tests which they should pass if they are to describe some of the hair of three charge black holes and three charge black rings.
\end{abstract}




\section{Introduction}

Recently, there has been a renewed interest in the properties of supertubes [1], due mainly to their possible use in counting black hole entropy. In the case of the D1-D5 system this has been successfully carried out [2, 3, 4, 5, 6, 7, 8, by relating the various configurations of two charge supertubes to the supersymmetric ground states of the D1-D5 system. Moreover, the supergravity solutions of these supertubes have been found to be regular. This gives a one to one map between microstates of the D1-D5 system and classical supergravity solutions, which can thus be thought of as being the hair of this system.

It is a rather fascinating possibility that this story extends to three charge black hole with a macroscopic horizon area. So far this has been directly checked only for a single unit of momentum [7]. Moreover, in [10] the three charge supertubes which might comprise the hair of the three charge black hole have been constructed ${ }^{1}$ using both the Born-Infeld action of the dipole branes (generalizing the construction in [1, 22]), and the nonabelian Born Infeld action of one of the constituent branes (generalizing the construction in [18, 19, 20]) - The properties of these three charge supertubes were found to be consistent with them describing some of the hair of the three charge black hole. Moreover, it was argued that three charge supertubes with angular momentum higher than that of the BMPV black hole should represent the hair of a yet to be found BPS three charge black ring.

It is important therefore to find the supergravity solutions for arbitrary three charge supertubes, and to see if these can be put in one-to-one correspondence with the states of the D1-D5-P system. So far these solutions have been hard to find using standard solution generating techniques; it seems like more powerful methods are needed.

Although in this paper we do not construct the most generic three charge supertube or BPS black ring solution, we construct the large radius (near tube) limit of these metrics, or otherwise put, the most generic metric sourced by a flat infinitely extended supertube. We hope in the near future to use these methods to find the full supertube and BPS black ring solutions.

The three charge supertubes constructed in [10] have F1, D0 and D4 charges, as well as D2, D6 and NS5 dipole moments. Their Born Infeld description can only capture the D2 and D6 dipole moments, but not the NS5 one. Thus, if wants to count three charge supertubes (in the way two charge supertubes have been counted [11]) it might not be enough to use the Born-Infeld action; one may have to count the BPS perturbations on the full near-tube geometry. Thus, finding this near-tube geometry is an important step towards the counting of the most generic three charge supertubes.

The crucial ingredient in our search for the near-supertube metric is the fact that the supersymmetries preserved by the three charge supertube background and those of the three charge black hole are the same. This allows one to use a few of the rather powerful methods to find supersymmetric solutions that have been developed over the past few years by Warner, Nemeschansky, Pilch and collaborators 9]. Rather than solving complicated the 2'nd order equations, supersymmetric solutions can be found by using the Killing spinors and their projectors to find algebraic first order relations between the metric and the forms, which are

\footnotetext{
${ }^{1}$ Other three charge supertubes have also appeared in [23].
} 
much easier to solve.

As we will see, tubes with three charges and three dipole charges come in two types. If the densities satisfy a certain relation, the geometry is smooth and has a zero size horizon. As we will argue in section 4, this solution is the near tube limit of the supertube metric. If the charge densities are higher, the metric has a horizon of nonzero area. This is nothing but the metric of the large radius limit of a BPS black ring. The existence of this black ring was conjectured [10] to account for the multiplicity of tubes with angular momentum larger than that of a BMPV black hole; this is a first confirmation of its existence.

Near a supertube with three charges and three dipole charges the metric is of the form $A d S_{3} \times S^{2} \times T^{6}$, in the duality frame in which the three charges are perpendicular M2 branes. The near horizon metric of the BPS black ring is of the form $A d S_{2} \times S^{2} \times S^{1} \times T^{6}$. Moreover, the Ricci scalar and other invariants are finite. Hence, both these geometries are smooth at $r=0$, and can be continued beyond this point. The fact that the geometry near the most generic three charge supertube is smooth is an important test which these supertubes must pass if they are to be the hair of the three charge black hole. We find that they indeed pass this test.

We also show that the horizon area of the black ring is zero when one supertube geometry exists, and starts increasing as the number of supertube configurations increases. The fact that a black ring with a nonzero horizon area appears exactly when the multiplicity of three charge supertube configuration becomes nonzero is a rather powerful confirmation of the picture of black hole microstates put forth in [7, 8]

In section 2 we find the flat supertube/black tube solution using the methods discussed above. In section 3 we discuss some of the special types of three charge supertubes, including the tube with three charges and two dipole charges discussed in [10]. In section 4 we discuss the properties of the most generic supertubes (with three charges and three dipole charge), as well as the properties of the near horizon limit of the BPS black ring. We also discuss how the multiplicity of supertube configurations increases with the horizon area of the corresponding black tube, and explore the implications of this for black hole physics. We conclude in section 5 .

While this work was being prepared for publication, we became aware of [12], where one of the metrics of the supertubes with three equal charges was found using the G-structure techniques of [13, 14] in 6 dimensional minimal supergravity. It would be interesting to see if those techniques can be used to find the metrics of the most generic three charge supertubes and black tubes whose near horizon limit we give here.

\section{The large radius limit of the three charge supertube solution.}

The three charge supertubes which should be some of the hair of the D1-D5-P black hole can be related by U-duality to the D0-D4-F1 supertubes found in [10], or to other configurations. In order to find the supergravity solution, it is useful to use a U-duality frame in which the symmetry between the three charges and the three dipole charges is manifest. Such a duality 
frame can be obtained by T-dualizing the configuration in [10] twice along two of the $T^{4}$ directions, and lifting to 11 dimensions. This gives three M2 brane charges, in the 123, 145, and 167 planes, as well as three M5 brane dipole moments, in the $12345 \vartheta, 14567 \vartheta$, and $12367 \vartheta$, directions. This duality frame has also the advantage of being free from the usual pathologies associated with boosting Kaluza Klein monopoles.

In the large radius/near supertube limit, the $\vartheta$ direction becomes infinitely extended - we henceforth call this direction $z$. The three M5 brane dipole moments become M5 brane charges, and the angular momentum in the $\vartheta$ direction becomes momentum in the $z$ direction. The polar coordinates on the three directions transverse to the tube are $r, \theta$ and $\phi$.

A generic two charge supertube can have a shape given by an arbitrary closed curve in the transverse space, and can also have arbitrary brane densities along this curve, as long as certain constraints are observed. The solutions we construct are for this curve being an infinite straight line, and for arbitrary local densities of the three charges. Thus, we allow the metric and forms to vary both along the tube (in the $z$ direction) and radially, but we require them to be $\mathrm{SO}(3)$ invariant.

We take the metric to have Lorentz frames of the form:

$$
\begin{aligned}
e^{1} & =e^{-2 A_{1}(r, z)-2 A_{2}(r, z)-2 A_{3}(r, z)}\left(d x_{1}+k(r, z) d z\right), \\
e^{2} & =e^{-2 A_{1}(r, z)+A_{2}(r, z)+A_{3}(r, z)} d x_{2}, \\
e^{3} & =e^{-2 A_{1}(r, z)+A_{2}(r, z)+A_{3}(r, z)} d x_{3}, \\
e^{4} & =e^{A_{1}(r, z)-2 A_{2}(r, z)+A_{3}(r, z)} d x_{4}, \\
e^{5} & =e^{A_{1}(r, z)-2 A_{2}(r, z)+A_{3}(r, z)} d x_{5}, \\
e^{6} & =e^{A_{1}(r, z)+A_{2}(r, z)-2 A_{3}(r, z)} d x_{6}, \\
e^{7} & =e^{A_{1}(r, z)+A_{2}(r, z)-2 A_{3}(r, z)} d x_{7}, \\
e^{8} & =e^{A_{1}(r, z)+A_{2}(r, z)+A_{3}(r, z)} d z \\
e^{9} & =e^{A_{1}(r, z)+A_{2}(r, z)+A_{3}(r, z)} d r \\
e^{10} & =e^{A_{1}(r, z)+A_{2}(r, z)+A_{3}(r, z)} r d \theta \\
e^{11} & =e^{A_{1}(r, z)+A_{2}(r, z)+A_{3}(r, z)} r \sin \theta d \phi
\end{aligned}
$$

The three M2 brane charges are in the 23,45 and 67 planes. In the flat tube limit of the metric, everything is a function of $r$ and $z$ only. The harmonic functions $Z_{i}$ which are commonly used to write this metric are

$$
Z_{i}=e^{6 A_{i}}
$$

Besides the electric 4-forms which we expect for smeared M2 branes, we also have a few magnetic 4-forms which correspond to the M5 charges of the supertube:

$$
\begin{aligned}
& F_{123 r}=-6 A_{1}^{(1,0)} e^{-6 A_{1}} \\
& F_{145 r}=-6 A_{2}{ }^{(1,0)} e^{-6 A_{2}} \\
& F_{167 r}=-6 A_{3}^{(1,0)} e^{-6 A_{3}}
\end{aligned}
$$




$$
\begin{aligned}
F_{123 z} & =-6 A_{1}{ }^{(0,1)} e^{-6 A_{1}} \\
F_{145 z} & =-6 A_{2}{ }^{(0,1)} e^{-6 A_{2}} \\
F_{167 z} & =-6 A_{3}{ }^{(0,1)} e^{-6 A_{3}} \\
F_{23 z r} & =2 t_{1} \\
F_{45 z r} & =2 t_{3} \\
F_{67 z r} & =2 t_{3} \\
F_{231011} & =2 d_{1} \sin \theta \\
F_{451011} & =2 d_{2} \sin \theta \\
F_{671011} & =2 d_{3} \sin \theta
\end{aligned}
$$

In the case of the 2-charge supertube, $A_{3}=d_{1}=d_{2}=t_{i}=0$. For no supertubes, $d_{i}=$ $t_{i}=k=0$. Since everything depends on $r$ and $z$, the Bianchi identities for the 4 -forms in equations (13) 211) are automatically satisfied. For $F_{i j} 1011$ these identities imply that $d_{1}, d_{2}$ and $d_{3}$ are constant.

For no supertube, each of the three M2 branes present breaks some of the supersymmetry. The 4 remaining Killing spinors are

$$
\epsilon_{i}=e^{-A_{1}-A_{2}-A_{3}} \eta_{i}
$$

where $\eta_{i}$ is a constant spinor annihilated by the three M2 brane projectors

$$
\left(1+\Gamma^{123}\right) \eta_{i}=\left(1+\Gamma^{145}\right) \eta_{i}=\left(1+\Gamma^{167}\right) \eta_{i}=0
$$

The crucial ingredient used in constructing this solution is the fact that the supertube preserves the same supersymmetry as its components. Therefore the above Killing spinors should also be Killing spinors of the supertube background. It is a straightforward exercise to use the supersymmetry variations, and find that in order for this to happen, the magnetic field strengths and $k$ should be related by:

$$
\begin{aligned}
-r^{2} t_{1} e^{6 A_{1}} & =d_{2} e^{6 A_{2}}+d_{3} e^{6 A_{3}}+3 r^{2} k A_{1}^{(1,0)}, \\
-r^{2} t_{2} e^{6 A_{2}} & =d_{1} e^{6 A_{1}}+d_{3} e^{6 A_{3}}+3 r^{2} k A_{2}^{(1,0)}, \\
-r^{2} t_{3} e^{6 A_{3}} & =d_{2} e^{6 A_{2}}+d_{1} e^{6 A_{1}}+3 r^{2} k A_{3}^{(1,0)}, \\
-r^{2} k^{(1,0)} & =2\left(d_{1} e^{6 A_{1}}+d_{2} e^{6 A_{2}}+d_{3} e^{6 A_{3}}\right)
\end{aligned}
$$

In principle the Bianchi identities and the supersymmetry transformations are enough to determine that the background is a solution. However, in the case of the usual D-branes these equations give a solution sourced by an arbitrary distribution of branes. To find the solution for empty space with at most point sources one must use some of the equations of motion as well.

The easiest equations of motion to use come from $d * F=F \wedge F$, and give three other equations:

$$
A_{1}^{(0,2)}+A_{1}^{(2,0)}=\frac{4 d_{2} d_{3} e^{-6 A_{1}}-6 r^{3} A_{1}^{(1,0)}-18 r^{4}\left(A_{1}^{(1,0)}\right)^{2}}{3 r^{4}}
$$




$$
\begin{aligned}
& A_{2}^{(0,2)}+A_{2}^{(2,0)}=\frac{4 d_{1} d_{3} e^{-6 A_{2}}-6 r^{3} A_{2}^{(1,0)}-18 r^{4}\left(A_{2}^{(1,0)}\right)^{2}}{3 r^{4}} \\
& A_{3}^{(0,2)}+A_{3}^{(2,0)}=\frac{4 d_{2} d_{3} e^{-6 A_{3}}-6 r^{3} A_{3}^{(1,0)}-18 r^{4}\left(A_{3}^{(1,0)}\right)^{2}}{3 r^{4}},
\end{aligned}
$$

which imply that the functions $Z_{i} \equiv e^{6 A_{i}}$ obey the equations:

$$
\begin{aligned}
& \frac{\partial^{2} Z_{1}}{\partial z^{2}}+\frac{\partial^{2} Z_{1}}{\partial r^{2}}+\frac{2}{r} \frac{\partial Z_{1}}{\partial r}=\frac{8 d_{2} d_{3}}{r^{4}} \\
& \frac{\partial^{2} Z_{2}}{\partial z^{2}}+\frac{\partial^{2} Z_{2}}{\partial r^{2}}+\frac{2}{r} \frac{\partial Z_{2}}{\partial r}=\frac{8 d_{1} d_{3}}{r^{4}} \\
& \frac{\partial^{2} Z_{3}}{\partial z^{2}}+\frac{\partial^{2} Z_{3}}{\partial r^{2}}+\frac{2}{r} \frac{\partial Z_{3}}{\partial r}=\frac{8 d_{2} d_{1}}{r^{4}} .
\end{aligned}
$$

The left hand side of this equation is nothing but the harmonic equation in four dimensions. For no supertubes, the right hand side of each of the three equations above is zero, and the metric is the one sourced by an arbitrary smeared distribution of the three types of M2 branes present.

In the case of a two charge supertube, two of the dipole moments are zero, so the functions $Z_{i}$ are again harmonic. The generic solution [21, 2, 6] is simply given by smearing these harmonic functions along the curves which determine the shape of the tube.

When two of the dipole charges are nonzero, the functions $Z_{i}$ are harmonic away from the tube; however near the tube the right hand side of (34- 36) becomes important. Therefore, this solution, as well as the other three charge supertube solutions cannot be obtained from harmonic brane solutions by usual solution generating techniques.

If the charge densities are independent on the $z$ direction, the equations above have very simple asymptotically flat solutions:

$$
\begin{aligned}
Z_{1} & =\frac{4 d_{2} d_{3}}{r^{2}}+\frac{Q_{1}}{r}+1 \\
Z_{2} & =\frac{4 d_{1} d_{3}}{r^{2}}+\frac{Q_{2}}{r}+1 \\
Z_{3} & =\frac{4 d_{2} d_{1}}{r^{2}}+\frac{Q_{3}}{r}+1 \\
\frac{\partial k}{\partial r} & =-\frac{2}{r^{2}}\left(d_{1} Z_{1}+d_{2} Z_{2}+d_{3} Z_{3}\right) \\
k & =+\frac{8 d_{1} d_{2} d_{3}}{r^{3}}+\frac{d_{1} Q_{1}+d_{2} Q_{2}+d_{3} Q_{3}}{r^{2}}+\frac{2 d_{1}+2 d_{2}+2 d_{3}}{r}
\end{aligned}
$$

When the charge densities have a nontrivial dependence along the tube directions, the solutions are more involved, and possibly quite interesting - among them might be for example a solution with several 3 charge black holes connected by a straight tube. We hope to explore those and other solutions in the future. 


\section{The physics of the solutions - special cases}

As we have seen, when only one dipole charge is present, the functions $Z_{i}$ are harmonic, which explains why the two charge supertube solutions found in [21, 2, 6] are given by smearing the source of the harmonic function over the curve which gives the tube shape.

When two or three of the dipole charges of the supertube are nonzero, the rotation parameter $k$ given by (301) is always such that $g_{z z}$ is zero near the branes. This also happens for the two charge supertubes, and should be expected, since the $z$ direction is common to the 3 M5 branes in the problem. .

Since the $z$ direction is the direction along the tube, and is periodically identified, if $g_{z z}$ becomes negative inside a region, then the solutions have closed timelike curves. The absence of these curves requires:

$$
k^{2} \leq Z_{1} Z_{2} Z_{3} .
$$

Like in the two charge supertube case, this relation determines a lower bound on the densities of the three charges.

In the following subsections we will be focusing on tubes where the local densities do not depend on $z$; hence the solution is given by (41).

\subsection{The two charge supertube - two charges and one dipole charge}

In this case

$$
Z_{3}=1, \quad Z_{1}=1+\frac{Q_{1}}{r}, \quad Z_{2}=1+\frac{Q_{2}}{r}, \quad k=\frac{2 d_{3}}{r}
$$

This reproduces the near tube form of the metric found in [21, 6]. The condition that the background be free from closed timelike curves implies $4 d_{3}^{2} \leq Q_{1} Q_{2}$. However, as we know from the D-brane physics of the tube, and as also found in the analysis of [6], the inequality must be saturated in order for the solution to be regular. Thus, the two charge tubes satisfy

$$
4 d_{1}^{2}=Q_{2} Q_{3} .
$$

If the charges are higher, the solution is that of coincident supertubes and regular branes, and is probably not a bound state.

\subsection{Three charges and two dipole charges}

The tubes with three charges and two dipole charges have been explored in [10] using the Born Infeld action. The tubes with a minimum number of dipole branes (which are the largest, and can be thought of as the building blocks of tubes with higher dipole charges) were found to have the property

$$
\frac{Q_{2}^{D}}{Q_{0}}=\frac{Q_{6}^{D}}{Q_{4}}
$$


where $Q_{0}$ and $Q_{4}$ are the local zero brane and 4-brane charge densities. It is quite remarkable that the same relation comes up if we require the near tube geometry to be free of closed timelike curves. Indeed, near the tube the leading terms in the harmonic functions are

$$
Z_{1} \approx \frac{4 d_{2} d_{3}}{r^{2}}, \quad Z_{2} \approx \frac{Q_{2}}{r}, \quad Z_{3} \approx \frac{Q_{3}}{r}
$$

and the leading terms in (41) are

$$
k \approx \frac{d_{2} Q_{2}+d_{3} Q_{3}}{r^{2}}
$$

Equation (42) is only satisfied when

$$
\left(d_{2} Q_{2}+d_{3} Q_{3}\right)^{2} \leq 4 d_{2} d_{3} Q_{2} Q_{3}
$$

which implies

$$
Q_{3} d_{3}=Q_{2} d_{2}
$$

Thus the background is free of closed timelike curves only when the tubes have the property (45), which also comes out naturally from the Born-Infeld analysis. The subleading terms in (42) also put a lower bound on the charge densities, similar to the ones in the two charge case. Equation (49) also implies that the local charge densities $Q_{2}$ and $Q_{3}$ cannot vary independently along the tube.

The Ricci scalar near the branes diverges like $r^{-2 / 3}$; however this probably signifies the presence of brane sources, and is not a pathology. In our U-duality frame the two charge supertube (which is regular in the D1-D5 duality frame [6]) has a Ricci scalar which diverges like $r^{-4 / 3}$, again signifying the presence of brane sources ${ }^{2}$.

\subsection{Three charges and one dipole charge}

In the Born Infeld analysis of [10] this case is pathological. Indeed, consider a D4 - F1 $\rightarrow$ D6 supertube, and try to add some D0 charge (the third charge) on the tube without turning on a D2 dipole moment. The D0 branes feel no force from the D4 branes and F1 strings, but are repelled by the D6 branes. Thus, a second dipole charge (D2 brane charge in this case) is needed to adhere the D0 branes to the D4-F1 supertube.

In the absence of a second dipole moment, the leading terms in the harmonic functions are

$$
Z_{i} \approx \frac{Q_{i}}{r}
$$

while

$$
k \approx \frac{Q_{1} d_{1}}{r^{2}}
$$

\footnotetext{
${ }^{2}$ This is not surprising; many D-brane solutions which have a divergent Ricci scalar are related by $\mathrm{T}$ duality to D3 brane solutions which are smooth.
} 
Thus, for small enough $r, k^{2}$ will always be bigger than $Z_{1} Z_{2} Z_{3}$, and so the background always has closed timelike curves. Therefore the tubes with three charges and only one dipole charge are not good solutions, as expected from the Born Infeld analysis.

We should also note that if one does not identify $z$ with an angular direction but keeps it infinitely extended, the closed timelike curves do not appear, and the metric is perfectly well behaved. The curvature does diverge near the tube, but this might be again the result of brane sources, and not a problem.

\section{The generic case - supertubes and black supertubes}

In the general case, when the three charges and three dipole charges are nonvanishing, the asymptotic form of the metric near the tubes is:

$$
d s^{2} \approx-\frac{r^{4}}{R_{0}^{4}} d t^{2}+2 \frac{r}{R_{0}} d t d z+c_{z} d z^{2}+\frac{R_{0}^{2}}{r^{2}} d r^{2}+R_{0}^{2}\left(d \theta^{2}+\sin ^{2} \theta d \phi^{2}\right)+d s_{T_{6}}^{2}
$$

where $R_{0} \equiv 2\left(d_{1} d_{2} d_{3}\right)^{1 / 3}$, the metric on the 6 -torus is flat, and

$$
\begin{aligned}
R_{0}^{4} c_{z} & =-16 d_{1}{ }^{2} d_{2} d_{3}-16 d_{1} d_{2}{ }^{2} d_{3}-16 d_{1} d_{2} d_{3}{ }^{2}+ \\
& +2 d_{1} d_{2} Q_{1} Q_{2}+2 d_{1} d_{3} Q_{1} Q_{3}+2 d_{2} d_{3} Q_{2} Q_{3}-d_{1}{ }^{2} Q_{1}{ }^{2}-d_{2}{ }^{2} Q_{2}{ }^{2}-d_{3}{ }^{2} Q_{3}{ }^{2}
\end{aligned}
$$

The metric has a horizon at $r=0$, and a transverse 2 -sphere of fixed radius $R_{0}$. The Ricci scalar at the horizon is constant, and proportional to the inverse square of the sphere radius.

$$
R_{\text {scalar }} \approx \frac{1}{2 R_{0}^{2}}
$$

Thus, for large $R_{0}$ the geometry is smooth. The physics of the solution depends crucially on the sign of $c_{z}$. If $c_{z}$ is negative, the background has closed timelike curves, and is nonphysical. This introduces a lower bound on the charge densities. As we will see in the next subsection, for $c_{z}=0$ the geometry is the large radius limit of the metric of a regular three charge supertube. For $c_{z}>0$, the horizon area is nonzero, and the geometry is that of a black string. This metric should be the large radius limit of the metric of a black supertube, which should reduce to a 5 dimensions BPS black ring.

\subsection{Regular three charge supertubes}

When $c_{z}=0$ the metric becomes

$$
d s^{2} \approx-\frac{r^{4}}{R_{0}^{4}} d t^{2}+2 \frac{r}{R_{0}} d t d z+\frac{R_{0}^{2}}{r^{2}} d r^{2}+R_{0}^{2} d \Omega_{2}^{2}+d s_{T_{6}}^{2}
$$

One can introduce new coordinates $u, v$ given by

$$
t=-u+v, \quad z=u+v
$$


and a new radial coordinate $r=\frac{\rho^{2}}{8 R_{0}}$. Ignoring subleading terms, the metric becomes

$$
d s^{2} \approx \frac{\rho^{2}}{4 R_{0}^{2}}\left(-d u^{2}+d v^{2}\right)+\frac{4 R_{0}^{2}}{\rho^{2}} d \rho^{2}+R_{0}^{2} d \Omega_{2}^{2}+d s_{T_{6}}^{2}
$$

Thus, the metric in the near tube region is locally $A d S_{3} \times S^{2} \times T^{6}$.

Since the $A d S$ metric can be continued smoothly past $r=0$, and neither the Ricci scalar nor other invariants diverge there, the metrics of these three charge configurations are completely smooth. This is an important test these solutions must pass if they are to represent the hair of the three-charge black hole, as conjectured in [7, 8].

The size of the $A d S_{3} \times S^{2}$ region is of order the Planck scale for small $d_{i}$, and larger for tubes with larger M5 dipole charges. Our brief investigation shows that this metric can be continued behind $r=0$, has at most a zero area horizon, no closed timelike curves, and no singularities. Thus, it has the right properties to describe a three charge supertube. There is another way to see that this metric should indeed describe a supertube: When $Q_{1}=Q_{2}=Q_{3}=Q$ and $d_{1}=d_{2}=d_{3}=d$, the condition $c_{z}=0$ becomes $Q^{2}=16 d^{2}$. This condition is very natural if we think about the three charge supertube as being made of three simple tubes with two charges. The charge densities of each tube are $Q / 2, Q / 2$. As we have seen in (44), a two charge tube has densities $Q_{i} Q_{j}=4 d_{k}^{2}$, which implies $Q^{2}=16 d^{2}$, and hence $c_{z}=0$. Thus, it appears that the $c_{z}=0$ is a natural property of the supertube with three charges and three dipole charges.

This solution is reminiscent of the M3 brane of [15], which is also made of three M5 branes preserving 4 supercharges [16], or of the M-theory $A d S$ solutions found in [17]. It would also be interesting to see if there exists a two dimensional conformal field theory dual to M-theory on this space.

\subsection{Black supertubes}

When $c_{z}$ is greater than zero, the area of the horizon at $r=0$ is larger than zero. Indeed, the horizon is extended in the $z, \theta, \phi$ directions, and $g_{z z} g_{\theta \theta} g_{\phi \phi}$ is finite at $r=0$. Thus, the metric for $c_{z}>0$ describes the large radius limit of a BPS black tube (which should descend to a black ring in 5 dimensions).

Near the horizon the metric is

$$
d s^{2} \approx-\frac{r^{4}}{R_{0}^{4}} d t^{2}+2 \frac{r}{R_{0}} d t d z+c_{z} d z^{2}+\frac{R_{0}^{2}}{r^{2}} d r^{2}+R_{0}^{2} d \Omega_{2}^{2}+d s_{T_{6}}^{2},
$$

which after completing the squares and ignoring subleading terms can be put in the form:

$$
d s^{2} \approx-\frac{r^{2}}{R_{0}^{2} c_{z}} d t^{2}+c_{z}\left(d z+\frac{r}{R_{0} \sqrt{c_{z}}} d t\right)^{2}+\frac{R_{0}^{2}}{r^{2}} d r^{2}+R_{0}^{2} d \Omega_{2}^{2}+d s_{T_{6}}^{2},
$$

Although it is not apparent, this metric is of the form $A d S_{3} \times S^{2} \times T^{6}$, with identifications coming from the fact that $z$ was originally an angular coordinate ${ }^{3}$. At first glance the horizon

\footnotetext{
${ }^{3}$ I thank Harvey Reall for pointing this out, and correcting a mistake in the previous version of this paper
} 
at $r=0$ is smooth, and the local $A d S$ coordinates can be extended behind the horizon in the usual way.

This black tube was conjectured to exist in [10], based on the understanding of the black hole microstates put forth in [7, 8]. Indeed, if small angular momentum supertubes are to be the hair of the BMPV black hole, it is but natural to expect that very large tubes, whose angular momentum is larger than the angular momentum of the BMPV black hole, represent the hair of another BPS black object, which should be a black ring.

The fact that we have found near-tube solutions with a nonzero horizon area is a rather compelling piece of evidence that such a black ring exists. We should note that this three charge BPS black ring would be the first of its kind, since other known black rings [24, 27, 26] are nonextremal, or have pathologies [25].

\subsection{Black Hole Physics}

For the purpose of discussing black hole physics it is convenient to look at tubes with three equal charges and three equal dipole charges $\left(Q_{1}=Q_{2}=Q_{3}=Q\right.$ and $\left.d_{1}=d_{2}=d_{3}=d\right)$ to make the arguments more transparent.

As we have also argued, it is very natural to assume that the relation $c_{z}=0$ is a fundamental one for a generic three charge supertube, much like the relation $\tilde{Q}_{1} \tilde{Q}_{2}=4 d^{2}$ is for a two charge supertube. In the equal charge case, these two relations are equivalent, and imply $Q=4 d$.

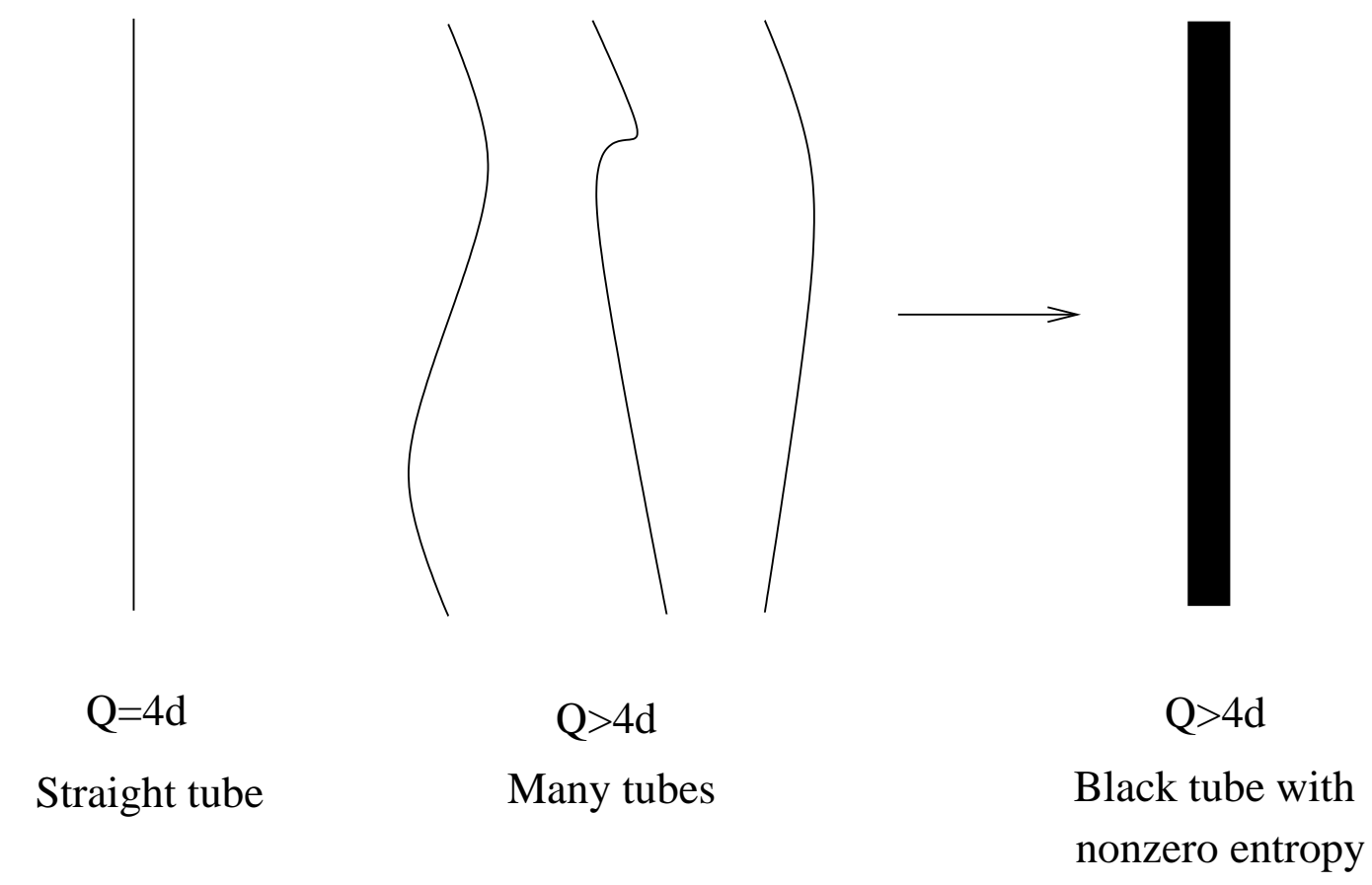

Figure 1: As one increases $Q$, the multiplicity of supertubes and the horizon area of the black tube increase. 
The picture which emerges from the above analysis is rather fascinating. If the local charge densities on a tube are such that $c_{z}=0$ (which in the equal charge case implies $Q=4 d$ ) the only spherically symmetric solution is a straight supertube. If the charge densities are higher, the only spherically symmetric solution is a black tube. However, we know from the Born Infeld analysis that supertubes do not have to be straight. Moreover, a wiggly supertube has the charge to dipole charge ratio higher than a straight tube. Thus, if $Q>4 d$ there are also quite a few regular supertube configurations where the tubes are wiggly. As $Q$ gets larger, the length of the regular tube one must fit in a fixed size box increases, and the tube can become more and more wiggly; hence, the number of configurations increases.

According to the interpretation of black hole horizons put forth in [7, 8], the microstates of a black object are regular geometries, and the horizon is the place where these microstate geometries start differing from each other. Moreover, the horizon area counts the number of these microstates.

As argued in [10], the microstates of the BPS black ring should be supertubes with angular momentum larger than that of the BMPV black hole. Therefore, in the large radius limit we discuss, the microstates of the black tube are wiggly supertubes. For $Q=4 d$, there is only one supertube configuration - the straight one. This is consistent with the horizon area of the black tube being zero. As $Q$ becomes larger than $4 d$, the tubes can become more and more wiggly, and their multiplicity increases. Moreover, their transverse size also increases. As the multiplicity and transverse size of the tubes increases, so does the horizon area of the black tube.

The fact that a black tube forms exactly when the multiplicity of states becomes nonzero, and that the horizon area and the multiplicity grow together is a spectacular confirmation of the picture of black hole microstates of [7, 8]. This is further strengthened by the fact that the transverse displacement of the wiggly tubes (hence the size of the region where they start differing from each other) also grows ${ }^{4}$. Hence, the supertube geometries do exactly what the hair of the black tube should do.

\section{Conclusions}

We have used some of the algebraic Killing spinor methods developed in [9] to find the large radius limit of the metric of three charge supertubes and BPS black rings. We have found that the supertubes with three charges and two dipole charges obey a constraint on the local charge densities. This constraint is identical to the one found in [10] via the Born-Infeld description of these supertubes.

We have also found that tubes with three charges and three dipole charges come in two types. If the charge densities and dipole charges satisfy a certain relation, the metric appears to be completely regular and there is no horizon - this metric is that of a straight supertube. If the charge densities are larger, a horizon of nonzero area forms, and the metric is the near horizon metric of a black ring.

\footnotetext{
${ }^{4}$ It would be interesting to try to find the geometries of the wiggly tubes and make this argument more precise.
} 
We have also showed that the horizon area of the black ring and the multiplicity of the supertube geometries grow together, much as one would expect if the supertube geometries were the hair of this black ring, as predicted in [10] based on the picture of black hole microstates of [7, 8].

There are quite a few directions to pursue. The first would be to use the algebraic Killing spinor methods used here to find the full metric of three charge supertubes and of three charge BPS black tubes. It is important to find these metrics, and to see if the most generic three charge supertube solutions are regular, and if they start differing from each other in a place where the horizon of the corresponding black hole would be. It would also be interesting to fully explore the structure of the metric near $r=0$, and to find its analytical continuation behind the horizon.

Equations (34 36) allow one to look for a few rather interesting generalizations of our solutions. For example it might be possible to construct a solution with several three black holes joined by a flat supertube, or a black tube solution with variable charge densities.

Perhaps the most important application of our solutions is to use them as a testing ground for the conjecture of [7, 8. Indeed, we have found a black tube whose horizon area is zero when there is only one supertube state, and increases as the number of supertubes increases. If one were able to explicitly count the three charge supertube configurations, (like Cabrera-Palmer and Marolf did in the two charge case [11]) and to explain the entropy of the black tube we would have a rather remarkable proof of the validity of this conjecture.

\section{Acknowledgements:}

I'm deeply indebted to Nick Warner for help with the algebraic Killing spinor methods used to find this solution. I thank Per Kraus and Don Marolf for excellent suggestions, and comments on the manuscript. I have benefitted from useful discussions with Radu Roiban, Henriette Elvang and Belkis Cabrera-Palmer, whom I would also like to thank for correcting a sign error in equations (27) 30). This work is supported in part by the NSF grant 0099590.

\section{References}

[1] D. Mateos and P. K. Townsend, "Supertubes," Phys. Rev. Lett. 87, 011602 (2001) arXiv:hep-th/0103030.

[2] O. Lunin and S. D. Mathur, "Metric of the multiply wound rotating string," Nucl. Phys. B 610, 49 (2001) arXiv:hep-th/0105136.

[3] O. Lunin and S. D. Mathur, "AdS/CFT duality and the black hole information paradox," Nucl. Phys. B 623, 342 (2002) arXiv:hep-th/0109154.

[4] O. Lunin and S. D. Mathur, "Statistical interpretation of Bekenstein entropy for systems with a stretched horizon," Phys. Rev. Lett. 88, 211303 (2002) arXiv:hep-th/0202072.

[5] O. Lunin, S. D. Mathur and A. Saxena, "What is the gravity dual of a chiral primary?," Nucl. Phys. B 655, 185 (2003) arXiv:hep-th/0211292. 
[6] O. Lunin, J. Maldacena and L. Maoz, "Gravity solutions for the D1-D5 system with angular momentum," arXiv:hep-th/0212210.

[7] S. D. Mathur, A. Saxena and Y. K. Srivastava, "Constructing 'hair' for the three charge hole," arXiv:hep-th/0311092.

[8] S. D. Mathur, "Where are the states of a black hole?," arXiv:hep-th/0401115.

[9] C. N. Gowdigere, D. Nemeschansky and N. P. Warner, "Supersymmetric solutions with fluxes from algebraic Killing spinors," arXiv:hep-th/0306097.

K. Pilch and N. P. Warner, "Generalizing the $\mathrm{N}=2$ supersymmetric RG flow solution of IIB supergravity," Nucl. Phys. B 675, 99 (2003) arXiv:hep-th/0306098.

K. Pilch and N. P. Warner, "N=1 supersymmetric solutions of IIB supergravity from Killing spinors," arXiv:hep-th/0403005.

D. Nemeschansky and N. P. Warner, "A family of M-theory flows with four supersymmetries," arXiv:hep-th/0403006.

I. Bena and N. P. Warner, in preparation

[10] I. Bena and P. Kraus, "Three charge supertubes and black hole hair," arXiv:hep-th/0402144.

[11] B. Cabrera - Palmer and D. Marolf, "Counting supertubes," arXiv:hep-th/0403025.

[12] O. Lunin, "Adding momentum to D1-D5 system," arXiv:hep-th/0404006.

[13] J. B. Gutowski, D. Martelli and H. S. Reall, "All supersymmetric solutions of minimal supergravity in six dimensions," Class. Quant. Grav. 20, 5049 (2003) arXiv:hep-th/0306235.

[14] J. P. Gauntlett and S. Pakis, "The geometry of D = 11 Killing spinors. ((T)," JHEP 0304, 039 (2003) arXiv:hep-th/0212008.

[15] M. Cvetic, G. W. Gibbons, H. Lu and C. N. Pope, "Supersymmetric M3-branes and G(2) Manifolds," Nucl. Phys. B 620, 3 (2002) arXiv:hep-th/0106026.

[16] I. Bena and M. Cvetic, unpublished.

[17] J. P. Gauntlett, D. Martelli, J. Sparks and D. Waldram, "Supersymmetric AdS(5) solutions of M-theory," arXiv:hep-th/0402153.

[18] I. Bena, "The polarization of F1 strings into D2 branes: 'Aut Caesar aut nihil'," Phys. Rev. D 67, 026004 (2003) arXiv:hep-th/0111156.

[19] W. I. Taylor, unpublished

[20] D. Bak and K. M. Lee, "Noncommutative supersymmetric tubes," Phys. Lett. B 509, 168 (2001) arXiv:hep-th/0103148. 
[21] R. Emparan, D. Mateos and P. K. Townsend, "Supergravity supertubes," JHEP 0107, 011 (2001) arXiv:hep-th/0106012.

[22] D. Mateos, S. Ng and P. K. Townsend, "Tachyons, supertubes and brane/anti-brane systems," JHEP 0203, 016 (2002) arXiv:hep-th/0112054.

[23] M. Kruczenski, R. C. Myers, A. W. Peet and D. J. Winters, "Aspects of supertubes," JHEP 0205, 017 (2002) arXiv:hep-th/0204103.

[24] R. Emparan and H. S. Reall, "A rotating black ring in five dimensions," Phys. Rev. Lett. 88, 101101 (2002) arXiv:hep-th/0110260.

[25] H. Elvang and R. Emparan, "Black rings, supertubes, and a stringy resolution of black hole non-uniqueness," JHEP 0311, 035 (2003) arXiv:hep-th/0310008.

[26] H. Elvang, "A charged rotating black ring," Phys. Rev. D 68, 124016 (2003) arXiv:hep-th/0305247.

[27] R. Emparan, "Rotating circular strings, and infinite non-uniqueness of black rings," arXiv:hep-th/0402149.

[28] H. S. Reall, "Higher dimensional black holes and supersymmetry," Phys. Rev. D 68, 024024 (2003) arXiv:hep-th/0211290. 